\title{
JUMP—From Java Annotations to UML Profiles ${ }^{\star}$
}

\author{
Alexander Bergmayr ${ }^{1}$, Michael Grossniklaus ${ }^{2}$, Manuel Wimmer ${ }^{1}$, and Gerti Kappel ${ }^{1}$ \\ 1 Vienna University of Technology, Austria \\ lastname@big.tuwien.ac.at \\ ${ }^{2}$ University of Konstanz, Germany \\ michael.grossniklaus@uni-konstanz.de
}

\begin{abstract}
The capability of UML profiles to serve as annotation mechanism has been recognized in both industry and research. Today's modeling tools offer profiles specific to platforms, such as Java, as they facilitate model-based engineering approaches. However, the set of available profiles is considerably smaller compared to the number of existing Java libraries using annotations. This is because an effective mapping between Java and UML to generate profiles from annotation-based libraries is missing. In this paper, we present JUMP to overcome this limitation, thereby continuing existing mapping efforts by emphasizing on annotations and profiles. We demonstrate the practical value of JUMP by contributing profiles that facilitate reverse-engineering and forward-engineering scenarios for the Java platform. The evaluation of JUMP shows that profiles can be automatically generated from Java libraries exhibiting equal or even improved quality compared to profiles currently used in practice.
\end{abstract}

Keywords: Java Annotations.UML Profiles·Model-Based Engineering.Forward Engineering.Reverse Engineering.

\section{Introduction}

Since the introduction of the UML profile mechanism, numerous profiles have been developed [38], many of which are available by the OMG standardization body [36]. Even in industry, the practical value of profiles has been recognized as today's modeling tools offer already predefined stereotypes covered by such profiles. They are considered as a major ingredient for current model-based software engineering approaches [6] by providing features supplementary to the UML standard metamodel. This powerful capability of profiles can also be exploited in terms of an annotation mechanism [42], where defined stereotypes show similar capabilities as annotations in Java. Hence, deriving stereotypes from established programming libraries to produce corresponding profiles at the modeling level is desirable. For instance, IBM's Rational Software Architect provides profiles for certain Java libraries. By applying such profiles, high-level platformindependent models (PIMs) are refined into models specific to a platform (PSMs), where the platform refers to the library from which the profile was derived. Turning this forward-engineering (FE) perspective into a reverse-engineering (RE) one, existing

* This work is co-funded by the European Commission under the ICT Policy Support Programme, grant no. 317859 . 
programs can be represented as UML models that capture annotations by applying the corresponding profiles. Therefore, platform-specific profiles and their application are beneficial from both perspectives. In a reverse-engineering step, model analyzers can exploit captured stereotypes to facilitate comprehension [10], whereas profiled UML models, i.e., models to which profiles are applied, pave the way for model transformers to generate richer program code in a forward-engineering step [42].

Problem. However, to date, an effective conceptual mapping between UML and Java as a basis for an automated process to generate profiles from libraries that use annotations is still missing. As a result, profiles need to be manually developed, which is only achievable by a huge effort when considering the large number of possible annotations in Java. In the ARTIST project [4], we are confronted with this problem, as we work towards a model-based engineering approach for modernizing applications by novel cloud offerings, which involves representing PSMs that refer to the platform of existing applications, e.g., the Java Persistence API (JPA), when considering persistence, and the platform of "cloudified" applications, e.g., the Objectify library ${ }^{1}$, when considering cloud datastores. For instance, JPA annotations at the modeling level facilitate distinguishing between plain association and composition relationships and precisely deciding on multiplicities, which is in general not easily to grasp [7]. UML models profiled by Objectify annotations enable generating method bodies even from a structural viewpoint. These examples highlight the practical value of platform-specific reverse-engineering and forward-engineering tools, which are developed in the ARTIST project.

Contribution. In this paper, we present a fully automatic transformation chain for generating UML profiles from Java libraries that use annotations. For that reason, we propose an effective conceptual mapping between the two technical spaces $[25,30]$. Thereby, we continue the long tradition of investigating mappings between Java and UML $[15,23,28,33]$. Though, in this work, we also consider Java annotations and UML profiles in the mapping process. This necessitates overcoming existing heterogeneities that, e.g., refer to the target specification of Java annotations and other peculiarities of how Java annotation types are declared. To operationalize the conceptual mapping, we employ model transformation techniques [12] as a basis for our approach JUMP, which allows developers to "jump" from annotation-based Java libraries to UML profiles. We collect all the automatically generated profiles and make them publicly available in terms what we call the UML-Profile-Store [43], thereby complementing OMG's collection of standardized profiles with supplementary profiles for the Java platform.

Structure. In Section 2, we motivate the practical value of platform-specific profiles by a typical JUMP use-case and we give the background for UML Profiles and Java Annotations in terms of metamodels. We present JUMP in Section 3 by providing insights into our proposed conceptual mapping and elaborating effective solutions to overcome existing heterogeneities of the two languages. In Section 4, we discuss our prototypical implementation based on the Eclipse ecosystem, while in Section 5, we evaluate $J U M P$. In particular, we $(i)$ compare our methodology how to represent annotations and annotation types in UML with methodologies used in current modeling tools and (ii) evaluate the quality of automatically generated profiles compared to profiles used in practice. Finally, in Section 6, we discuss related work and conclude in Section 7.

${ }^{1}$ https://code.google.com/p/objectify-appengine 


\section{Motivation and Background}

To motivate the practical value of platform-specific profiles, we introduce a typical JUMP use-case. Then, we discuss the concepts of Java's annotation mechanism and briefly introduce UML's profile mechanism to establish the basis for our approach.

\subsection{Application of Platform-Specific UML Profiles}

A typical JUMP use-case is directed to scenarios in the context of reverse-engineering (RE) and forward-engineering (FE). They are of particular relevance for migration projects, which aim at reinterpreting existing reengineering processes [26] in the light of advanced model-based engineering approaches [17]. In this respect, UML profiles play an important role as they enable models annotated with platform-specific information [39]. To demonstrate a concrete use-case, we selected the JPA and Objectify profile from the area of data modeling. The idea is to replace the former profile by the latter one, thereby realizing a change of the data access platform as typically required by "moving-to-the-cloud" scenarios. Figure 1 depicts an excerpt of the PSMs of a typical eCommerce web application, where the platform refers to the selected profiles. From the JPA-based PSM, a sliced PIM is generated that sets the focus solely on the domain classes, i.e., annotated with JPA stereotypes, which are intended to be modified. Even better, this generated PIM interprets JPA stereotypes in terms of native UML concepts. As a result, the accuracy of the PIM is improved because it explicitly captures identifiers, compositions, and more precise multiplicities. These improvements of the PIM demonstrate the practical value of considering platform-specific information in the context of a model-based RE scenario. Furthermore, they leverage the refinement

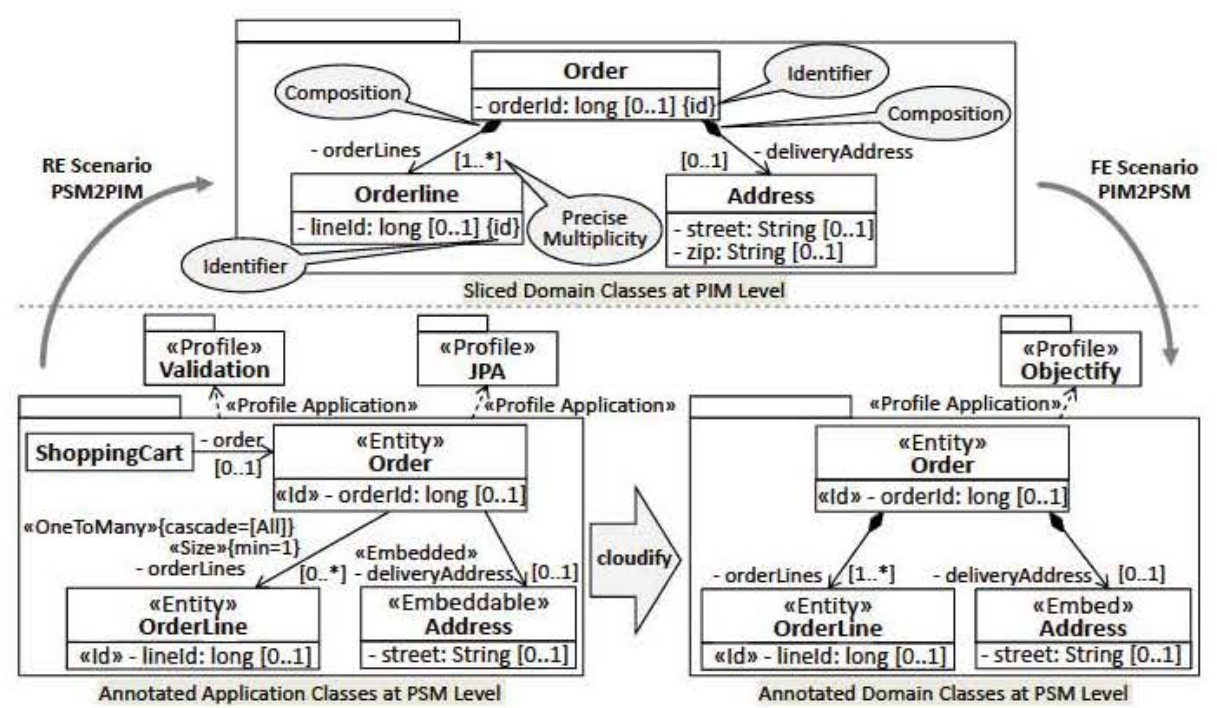

Fig. 1. Typical JUMP Use-Case 
of the PIM towards an Objectify-based PSM without the need to identify mappings between the pertinent platforms. From the produced Objectify-based PSM, program code can be generated by also interpreting applied stereotypes in the context of a FE scenario. For instance, method bodies for CRUD operations can be generated for domain classes as they are indicated by the respective stereotypes and generated code elements can be automatically annotated. Clearly, JUMP acts as an enabler for both RE and FE scenarios by providing the required platform-specific profiles.

\subsection{Mechanisms for Annotations in Java and Profiles in UML}

Before annotations can be applied on code elements, they need to be declared in terms of annotation types. A rough overview of the main concepts behind annotations in Java is given in the metamodel depicted in Figure 2a. We extracted this metamodel from the JLS7 [37]. AnnotationTypes declare the possible annotations for code elements and may have, similar to Java interface declarations, optional modifiers. They are identified by a name. AnnotationTypes may themselves be subject for annotations. Most importantly for the context of this work is the target annotation that is represented in the metamodel as an attribute for simplicity reasons. It indicates the code elements that are valid bases for an application of an AnnotationType. The body of an annotation type declaration consists of zero or more AnnotationTypeElements for holding information of AnnotationType applications. They are declared in terms of method signatures with optional modifiers, a mandatory type and name, and an optional default value that is returned if no custom value is set.

With the introduction of UML 2, the profile mechanism has been significantly improved compared to the beginnings of UML [18]. In particular, a profile modeling language has been incorporated in the UML language family to precisely define how profiles are applied on UML models. Figure $2 b$ depicts the core elements of UML's Profiles package and relates them to the Classes package of UML. As the Stereotype metaclass specializes the Class metaclass, it inherits modeling capabil-
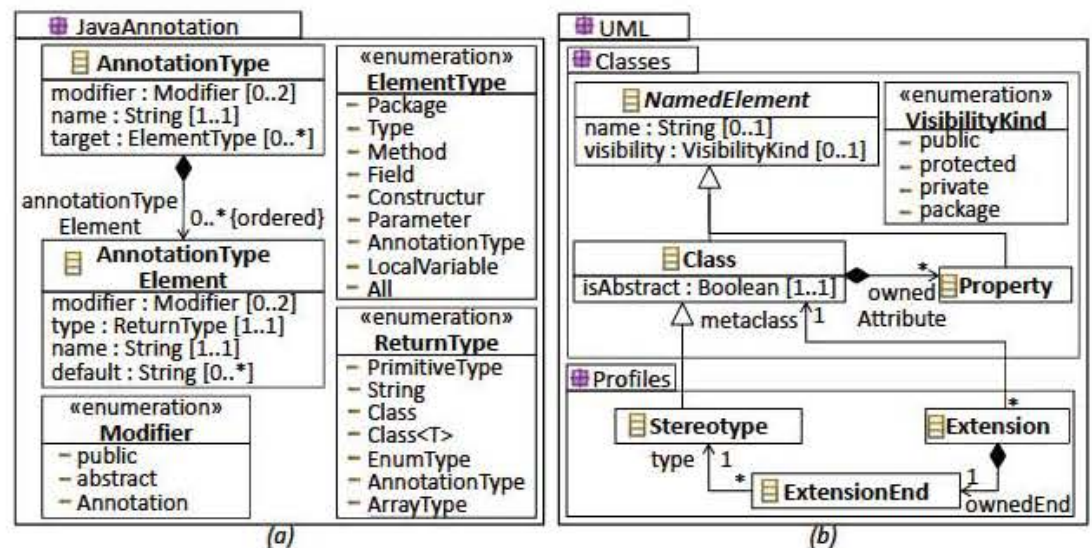

Fig. 2. Metamodel of Java Annotations and UML Profiles 
ities such as properties. Defined stereotypes reference the metaclasses that are extended by the Extension relationships. The ExtensionEnd realizes the reference from the extended metaclass back to the Stereotype. Similar to AnnotationTypes, Stereotypes are identified by a name property, and modified by an optional visibility and the mandatory isAbstract property.

To demonstrate the relationship between annotations and stereotypes, we set the focus on the Order class of the JPA-based PSM in Figure 1. Listing 1.1 shows the application of the Entity annotation type to the Order class whereas Listing 1.2 depicts the respective declaration at the programming level.
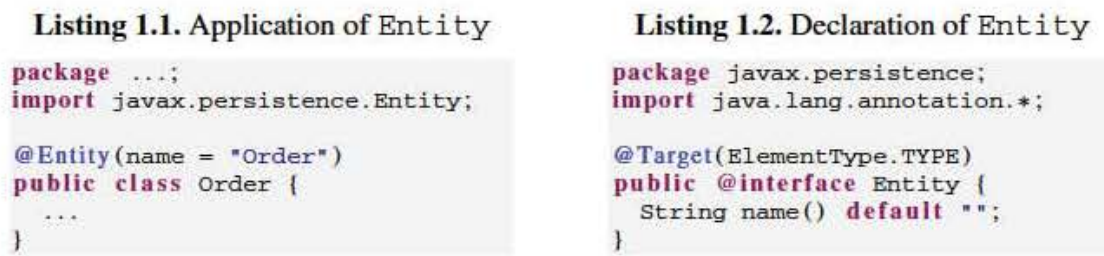

The corresponding UML-based representation is presented in Figure 3, which demonstrates the stereotype application to the Order class and the Entity declaration by a Stereotype. Similarly, at the package-level, the UML profile, which covers the Entity stereotype needs to be applied to the Order's package as a prerequisite for the stereotype application. To ensure that the Entity stereotype provides at least similar capabilities as the corresponding annotation type, the extension relationship references the UML metaclass Type. Furthermore, the stereotype comprises a property corresponding to the annotation type element name of the Entity.

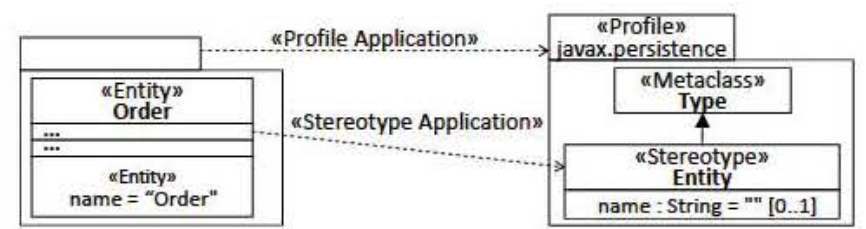

Fig. 3. Application and Definition of Entity Stereotype

\section{UML Profile Generation from Annotation-Based Java Libraries}

We start our investigation for generating UML profiles from annotation-based Java libraries by presenting the process of JUMP, as shown in Figure 4 . The entry-point to JUMP is Java Code that is translated into a corresponding Code Model, which is considered as a one-to-one representation of Java Code, i.e., the transition from a text-based to a model-based representation expressed in terms of MOF [35]/EMF [14]. The Code Model is the basis for generating a UML Profile, which facilitates to capture Java annotation type declarations in terms of UML stereotypes (cf. middle of Figure 4). In turn, they serve as foundation to apply profiles as an annotation mechanism [42]. In case of 


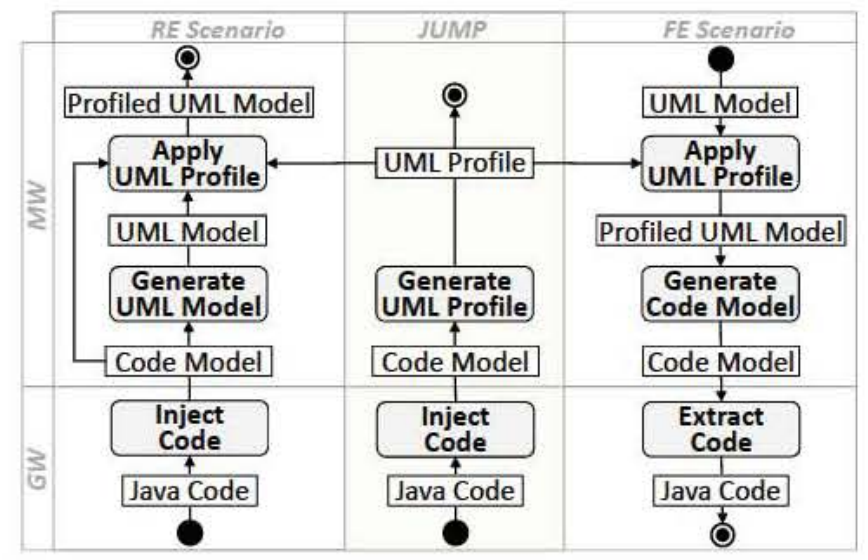

Fig. 4. Process for UML Profile Generation and their Application

reverse-engineering Java Code (cf. left hand side of Figure 4), the Profiled UML Model results from applying profiles to the generated UML Model, where the Code Model covers the annotated elements that indicate to which elements of the UML Model the corresponding stereotypes are applied. Similarly, in case of forward-engineering Java Code (cf. right hand side of Figure 4), profiles are applied to the UML Model even though, in this case, the Profiled UML Model serves as input for generating the Code Model from which Java Code is extracted. Bridging the two technical spaces [25] we are confronted with, i.e., GrammarWare (GW) [27] and ModelWare (MW) [30], is required for the two scenarios as well as JUMP.

\subsection{Bridging Technical Spaces}

Transforming plain Java code into a UML-based representation requires overcoming the different encoding and resolving language heterogeneities. Concerning the first aspect, the Java code needs to be encoded according to the format imposed by the modeling environment [5]. Concerning the second aspect, a bridge between Java and UML based on translations requires a conceptual mapping between the two languages. Instead of directly translating plain Java code into a UML-based representation, the use of a twostep approach is preferable [24], which is also applied by JUMP. In a first step, Java Code is translated into a Code Model that uses Java terminology and structures conforming to the Java metamodel provided by MoDisco [9]. This Code Model is the basis for generating UML profiles and input for the second step that is dedicated to resolving language heterogeneities by relying on the correspondences between the Java and UML metamodels.

\subsection{Generating UML Profiles}

To facilitate the generation of UML profiles, we present a conceptual mapping between Java's annotation concept and the concept of profiles in UML. Thereby, stereotypes play 
a vital role for representing annotation types at the modeling level as they enable their application in a controlled UML standard-compliant way. From a language engineering perspective, stereotypes only extend the required UML metaclasses and facilitate defining constraints and model operations, such as model analysis or transformations, because they can directly be used in terms of explicit types similar to a metaclass in UML. Our proposed mapping is generic in the sense that any declared annotation type can be represented by a stereotype.

Table 1. Mappings between Java Annotations and UML Profiles

\begin{tabular}{|c|c|}
\hline Java Concept & UML Concept \\
\hline AnnotationType a & add Stereotype s \\
\hline a.name & s.name $=$ a.name \\
\hline a.annotationTypeElement & add Property $\mathrm{p}$ for each AnnotationTypeElement in a.annotationTypeElement \\
\hline \multicolumn{2}{|l|}{ switch(a.modifier) } \\
\hline case : public & s.visibility = public \\
\hline case : abstract & s. isAbstract $=$ false \\
\hline $\begin{array}{r}\text { case : annotation an and } \\
\text { not an.type = Target }\end{array}$ & apply Stereotype for an.type to $\mathrm{s}$ \\
\hline $\begin{array}{c}\text { case : annotation an and } \\
\text { an.type = Target }\end{array}$ & $\begin{array}{l}\text { add Property } p \text { for each ElementType in a.target } \\
\text { p.name = "base_".concat(p.type) } \\
\text { add Extension e for each ElementType in a.target } \\
\text { e.metaClass = p.type } \\
\text { add ExtensionEnd } f \\
\text { f.type }=s\end{array}$ \\
\hline \multicolumn{2}{|l|}{ switch(a.target) } \\
\hline case : AnnotationType & p.type = Stereotype \\
\hline case : Constructor & $\begin{array}{l}\text { p.type = Operation } \\
\text { add Constraint }\{\text { self.base_Operation.ocllsDefined() implies } \\
\text { self.base_Operation.name = } \\
\text { self.base_Operation.oclContainer().oclAsType(uml::Classifier).name\} }\end{array}$ \\
\hline case : Field & p.type $=\{$ EnumerationLiteral, Property $\}$ \\
\hline case : LocaleVariable & p.type $=$ Property \\
\hline case : Method & $\begin{array}{l}\text { p.type }=\{\text { Operation, Property }\} \\
\text { add Constraint }\{\text { self.base_Property.ocllsDefined() implies } \\
\text { self.base_Property.oclContainer().ocllsTypeOf(uml::Stereotype)\}}\end{array}$ \\
\hline case : Package & p.type $=$ Package \\
\hline case : Parameter & p.type $=$ Parameter \\
\hline case : Type & $\begin{array}{l}\text { p.type = Type } \\
\text { add Constraint \{self.base_Type.ocllsDefined() implies } \\
\text { Set\{uml::Stereotype,uml::Class,uml::Enumeration,uml::Interface\} -> } \\
\text { includes(self.base_Type.oclType())\} }\end{array}$ \\
\hline case : none & -- no Property $p$ needed \\
\hline case : all & $\begin{array}{c}\text { p.type }=\{\text { Class, Enumeration, Interface, Operation, Package, } \\
\text { Parameter, Property, Stereotype }\}\end{array}$ \\
\hline AnnotationElementType a & add Property $p$ \\
\hline a.name & p.name $=$ a.name \\
\hline a.default & p.default $=$ a.default \\
\hline \multicolumn{2}{|l|}{ switch(a.modifier) } \\
\hline case : public & p.visibility = public \\
\hline case : abstract & -- no corresponding feature \\
\hline case : annotation an & apply Stereotype for an.type to $p$ \\
\hline \multicolumn{2}{|l|}{ switch(a.type) } \\
\hline case : PrimitiveType & p.type = uml::PrimitiveType for a.type \\
\hline case : Class & p.type = uml::Class \\
\hline case : Class $\langle\mathrm{T}>$ & $\begin{array}{l}\text { p.type }=\text { uml::Class } \\
\text { apply javaProfile::JGenericType Stereotype to } p\end{array}$ \\
\hline case : EnumType & p.type = uml::Enumeration \\
\hline case : AnnotationType & p.type = uml::Stereotype \\
\hline case : ArrayType & -- infer lower and upper bound multiplicities \\
\hline
\end{tabular}


AnnotationType $\rightarrow$ Stereotype. The mapping presented in the upper part of Table 1 serves as a basis to generate a Stereotype from an AnnotationType. Thereby, not only its signature needs to be considered but also Java's Target meta-annotation. It determines the set of code elements an annotation type is applicable to. The name and, with two exceptions, the defined modifiers of an AnnotationType can straightforwardly be mapped to UML. First, the abstract modifier would lead to Stereotypes that cannot be instantiated if directly mapped. The problem is caused by Java's language definition. Although the abstract modifier is supported to facilitate one common type declaration production rule, it does not restrict the application of Annotationtypes. To ensure the same behavior on the UML level, we never declare a Stereotype to be abstract. Second, because annotations are considered as modifiers, it needs to be ensured that the Target annotation is properly treated. In fact, the defined set of Java ElementTypes determines the required set of Extensions to UML meta-classes that specify the application context of the stereotypes.

Generally, most Java ElementTypes correspond well to one or more UML metaclasses. Still, constraints are required for some ElementTypes to precisely restrict the application scope of the generated Stereotype according to their intention. UML does not explicitly support a constructor meta-class. The workaround is to map the Constructor to Operation and introduce a constraint that emulates the naming convention for constructors in Java. Note that annotation types can have several target types. Thus, before validating the OCL constraint, we have to check which target is actually used in the application. Similarly, the mapping of Java methods to UML requires a constraint as a declared method of an AnnotationType, i.e., AnnotationTypeElement, is mapped to a Property rather than an Operation in UML. This is because such methods do not provide a custom realization but merely return their assigned value when they get called. Properties in UML provide exactly this behavior. Hence, the constraint ensures that stereotypes generated from annotation types that target Java methods are applicable also to Property if they are contained by a Stereotype. Finally, we use a constraint to overcome the heterogeneity of Java's and UML's scope of Type. Consequently, stereotypes that extend Type are constrained to those elements that correspond to the set of elements generalized by Java's Type: AnnotationType, Class, Enumeration and Interface. The clear benefit of this approach is a smaller number of generated extension relationships between stereotypes and meta-classes in the profile.

AnnotationTypeElement $\rightarrow$ Property. AnnotationTypeElements are mapped to Properties as depicted in the lower part of Table 1. Except for the fact that UML properties cannot be defined as abstract, AnnotationTypeElements straightforwardly correspond to Properties. As AnnotationTypes in Java cannot explicitly inherit from super-annotations, the abstract modifier is rarely used in practice. To fully support all return types of AnnotationTypeElements, we introduce a Stereotype to properly address the fact that java. lang. Class provides generic capabilities, which is not the case for UML's meta-class Class. Hence, we apply our custom JGenericType stereotype to properties with return type Clas $<\mathrm{T}>$. 


\section{Implementation and Collected Profiles}

To show the feasibility of $J U M P$, we implemented a prototype based on the Eclipse ecosystem. We developed three transformation chains - JavaCode2UMLProfile, JavaCode2ProfiledUML, and ProfiledUML2JavaCode-to realize JUMP and the RE and FE scenarios introduced in Figure 1. For injecting Java Code, we employed MoDisco [9]. Hence, JUMP can be considered as a model discoverer to extract UML profiles from Java libraries. To realize the FE scenario, we extended the Java-based transformer provided by Obeo Network ${ }^{2}$. The prototype and the collection of profiles that we have generated for the evaluation of JUMP is available at the UML-Profile-Store [43]. It covers 20 profiles, comprising in total over 700 stereotypes. To share these profiles with existing community portals, we submitted them also to ReMoDD [16].

\section{Evaluation}

The evaluation of JUMP is twofold. First, we compare it with existing modeling tools regarding their representational capabilities for dealing with the declaration and application of Java annotation types. Second, we compare UML profiles automatically generated by JUMP with UML profiles delivered by IBM's Rational Software Architect. Thereby, our focus is on estimating the quality of the generated UML profiles.

\subsection{Methodological Evaluation}

As several commercial and open-source modeling tools provide modeling capabilities for UML and the Java platform, the aim of this study is to investigate on their methods for dealing with the application and declaration of annotations. For that reason, we set the focus on a Java-based reverse-engineering example that includes annotations and their declarations. We aim to answer the following research question $(R Q 1)$.

RQ1: What are the methods of current modeling tools to represent Java annotation types and their applications in UML and what are the practical implications?

To answer $R Q 1$, we define a set of comparison criteria that mainly address $(i)$ how the conceptual mapping between Java and UML for annotations is achieved by current modeling tools and $(i i)$ the generative capabilities of these tools regarding profiles. Based on the defined criteria, we evaluate six representative modeling tools and JUMP.

Comparison Criteria. As there are different approaches on how annotation types and their applications are represented at the modeling level, the first and the second comparison criteria ( $C C 1$ and $C C 2$ ) refer exactly to these extensional capabilities. The third criterion $(C C 3)$ refers to the support of generative capabilities regarding profiles.

$-C C 1$ : How are Java annotations applied to UML models?

$-C C 2$ : How are Java annotation type declarations represented in UML?

$-C C 3$ : Is the generation of UML profiles from Java code supported?

Selected Tools. We selected six major industrial modeling tools that claim to support reverse engineering capabilities for Java and UML, as summarized in Table 2.

Evaluation Procedure. We defined a simple reference application [43] that declares

${ }^{2}$ http://marketplace.eclipse.org/content/uml-java-generator 
a Java class to which we applied an annotation type from an external library. For the purpose of importing the application, we activated the offered functionality of the modeling tools required for a reverse-engineering scenario from Java to UML. While some of the modeling tools are delivered with standard configurations, other modeling tools allow configurations to change the reverse-engineering capabilities by using specific wizards. Moreover, some modeling tools go one step further and allow modifications on the transformation scripts used for the import of Java code. We evaluated the capabilities of the modeling tools offered in the standard settings and explored the different wizard configurations if supported, but we restrained from modifying transformation scripts.

Results. The results of our comparison are summarized in Table 2. Regarding the mapping between Java annotations and UML, we identified that the investigated modeling tools apply one of three significantly different approaches: $(i)$ annotations are considered as a built-in feature of the modeling tool, $(i i)$ a generic profile for Java is provided, which enables capturing annotations and their type declarations, and (iii) profiles are offered, which are specific to a Java library or even an application with custom annotation type declarations. Modeling tools with built-in support for annotations allow their application to arbitrary elements and so to UML elements. Clearly, such an approach facilitates to capture Java annotations, though the type declaration of the annotation in terms of a UML element and its application are not connected. The genericity of this approach, which goes beyond UML models, is clearly one reason for such a behavior. Providing a generic profile for Java means that the modeling tool emulates the representational capabilities of Java, which includes annotations. Although with this approach, the connection of annotation type declarations and their applications can be ensured, the native support of UML for annotating elements with stereotypes is still neglected. However, explicitly defined stereotypes for declared annotation types facilitate their reuse in a UML standard-compliant way and allow model operations to directly exploit them. With specific profiles for Java annotation types, these drawbacks can be overcome. While all evaluated modeling tools provide support for generating profiled UML class diagrams, none of them is capable of generating profiles from Java code.

Table 2. Comparison Results

\begin{tabular}{|c|c|c|c|c|c|}
\hline \multirow{2}{*}{\multicolumn{3}{|c|}{ Modeling Tool }} & \multicolumn{2}{|c|}{ Mapping (Java -> UML) } & \multirow{3}{*}{$\begin{array}{l}\text { UML Profile } \\
\text { Generation }\end{array}$} \\
\hline & & & Annotation & Annotation & \\
\hline Name & Version & Availability & Application & Declaration & \\
\hline $\begin{array}{l}\text { Visual Paradigm } \\
\text { www.visual-paradigm.com }\end{array}$ & 10.2 & $\begin{array}{l}\text { commercial } \\
\text { free community edition }\end{array}$ & Built-in Tool Feature & Class & - \\
\hline $\begin{array}{l}\text { Rational Software Architect } \\
\text { www.ibm.com/developerworks/rational/products/rsa }\end{array}$ & 8.5 .1 & $\begin{array}{l}\text { commerical } \\
\text { free for academice use }\end{array}$ & Specific Profiles & Stereotype & - \\
\hline $\begin{array}{l}\text { Magic Draw } \\
\text { www.nomagic.com }\end{array}$ & 17.0.4 & $\begin{array}{l}\text { commerical } \\
\text { free trial version }\end{array}$ & Generic Java Profile & Interface & - \\
\hline $\begin{array}{l}\text { Enterprise Architect } \\
\text { www.sparxsystems.com }\end{array}$ & 9.3 & $\begin{array}{l}\text { commerical } \\
\text { free for academice use }\end{array}$ & Built-in Tool Feature & Interface & - \\
\hline $\begin{array}{l}\text { Altova UML } \\
\text { www.altova.com/umodel.html }\end{array}$ & 2013 & $\begin{array}{l}\text { commerical } \\
\text { free for academice use }\end{array}$ & Generic Java Profile & Interface & - \\
\hline $\begin{array}{l}\text { ArgoUML } \\
\text { argouml.tigris.org }\end{array}$ & 0.34 & open-source & Generic Java Profile & Interface & - \\
\hline JUMP & 1.0 .0 & | open-source & Specific Profiles & Stereotype & + \\
\hline
\end{tabular}




\subsection{Quality Evaluation}

As UML profiles are already offered by current modeling tools, the aim of this study is to investigate their quality in comparison with profiles automatically generated by JUMP. For that reason, we conducted a positivist case study [32] based on real-world Java libraries to evaluate the commonalities and differences between generated profiles and profiles used in practice by following the guidelines of Roneson and Hörst [41]. In this study, we aim to answer the following research question $(R Q 2)$.

RQ2: How is the quality of UML profiles automatically generated from annotationbased Java libraries compared to UML profiles used in practice?

To answer $R Q 2$, we define the requirements of the case study, briefly mention the used Java libraries, and specify the measures based on which the comparison is conducted. Then, we discuss the results of our study not only from a syntactic perspective, but also from a semantic one. The rationale behind this two-step approach is that even though a syntactical matching process for comparing the profiles provides already valuable results, some interesting correspondences may still be uncovered because of potential syntactical and structural heterogeneities [46] between the compared profiles and the conservative matching strategy applied for the syntactical comparison.

Case-Study Design. To conduct this study, the source code of Java libraries that exploit annotations is required. Furthermore, we require existing profiles that claim to support the selected Java libraries at the modeling level. To accomplish an appropriate coverage of different scenarios, the selected Java libraries ideally comprise different intrinsic properties with respect to the design complexity and exploited language elements. Unfortunately, profiles specific to Java libraries in reasonable quality are rarely available. Consequently, in the process of selecting the Java libraries for this study, we were also confronted with the actual offering of modeling tools. IBM's Rational Software Architect (RSA) is obviously close to JUMP and offers several profiles of well-known Java libraries mainly for code generation purposes. Thus, we conducted this study by relying on profiles of RSA in version 8.5.1. We selected four established Java libraries for which the source code is available and a corresponding RSA profile in the same major version is offered: Java Persistence API (JPA), Enterprise Java Beans (EJB), Struts and Hibernate. RSA offers them in a UML standard-compliant way. Consequently, we could directly compare them without an intermediate conversion step. All the case-study data including the Java libraries and the profiles are available at our project web site [43].

Case-Study Measures. The measures used in the case study are based on model comparison techniques [29]. Thus, we are interested in equivalent elements that reside in our generated profiles and in the RSA profiles, elements that reside in both solutions but still show differences in their features, and elements that are only available in one of the compared solutions. The measures for estimating the quality of the generated profiles are collected in a two-step matching process. While the first step automatically collects measures based on syntactic model comparison, the second step relies on manually processing differences produced in the first step to deal with semantic aspects.

In the syntactic model comparison, we compute the following measures for certain model elements. To determine element correspondences, we employ as matching heuristic name equivalence, i.e., only if two elements have completely the same 
name, they are considered to be corresponding. If an element has no name, such as the Extension relationship, it is considered that the elements are corresponding if their source and target elements correspond. Finally, fine grained comparison of the feature values for the given elements is performed. Regarding model elements, we set the focus on (i) Stereotypes that are common to both and unique either to JUMP or RSA, (ii) differences regarding the Extensions of common Stereotypes, and (iii) differences regarding the Properties such Stereotypes cover.

In the semantic model comparison, we take the syntactical differences as input and aim at finding additional correspondences between elements which are hardly explored by a pure syntactic comparison due to the conservative matching strategy. We investigate unmatched elements, especially stereotypes, in our generated profiles and in the RSA profiles, and reason about possible element correspondences beyond String equivalences. Finally, in the semantic processing, we further evaluate the correspondences found in the first phase due to the potential syntactical and structural heterogeneities.

Results. We now present the results of applying JUMP to the four selected Java libraries and compare them to the profiles offered by RSA. The full results are also available at our project web site [43]. The absolute number of generated stereotypes by JUMP and the provided ones by RSA are depicted in Figure 5a. Figure 5b summarizes $(i)$ the number of stereotypes generated by JUMP but not covered by the RSA profiles, $(\mathrm{ii})$ the number of stereotypes that are exclusively covered by the RSA profiles, and ( $\mathrm{iii}$ ) the number of stereotypes that are common to both. These results include correspondences between stereotypes detected throughout the syntactic and semantic comparison. For instance, the EJB profile of RSA covers stereotypes that refer to the ELocal and @Remote annotations of the EJB library, though their signature
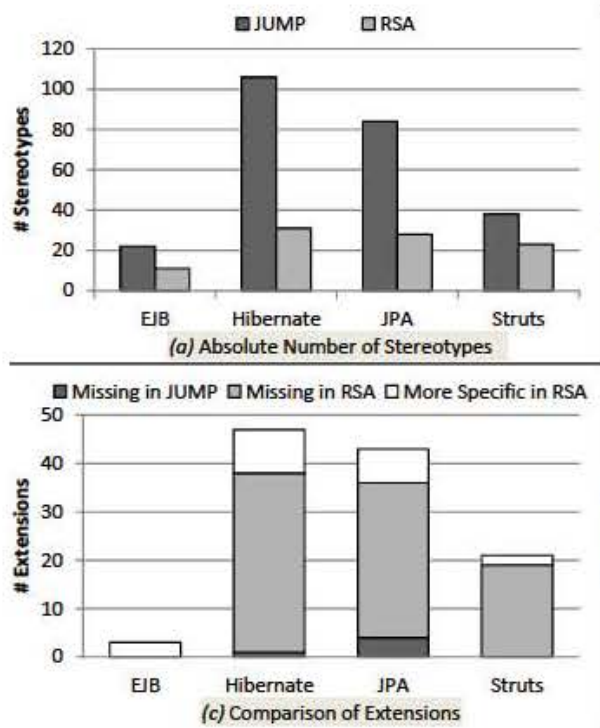
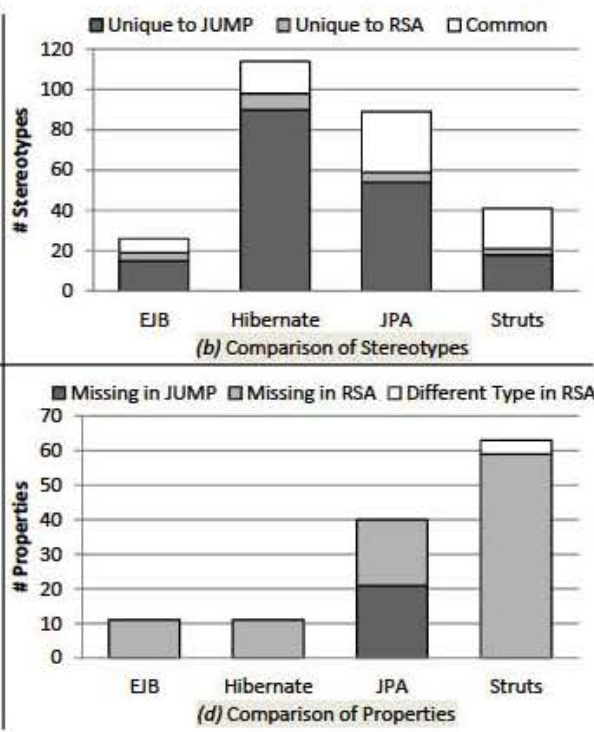

Fig. 5. Results of Quality Evaluation 
additionally contains the substring "Interface". Another example refers to the class QueryHint in the JPA profile of RSA, which is in fact an annotation type in the JPA library. In our solution, the QueryHint is represented by a stereotype even though it is also valid to use a class instead, because the QueryHint can not actually be applied, but can rather only be used inside of another annotation. Although some stereotypes in the set of common ones show differences regarding the meta-classes they extend, we granted them to be equal if the extended meta-classes are related by a generalization relationship. We encountered this case in the EJB and the JPA library with respect to extensions of the meta-classes Type and Class. Stereotypes generated by JUMP extend the more general meta-class Type because the scope of Java's element type Type also covers Enumeration, Interface and AnnotationType in addition to Class.

The comparison regarding extensions of stereotypes common to both JUMP and RSA is summarized in Figure 5c. In a few cases, the RSA profiles comprise extensions to the UML meta-class Association to allow stereotypes on associations between elements rather than on properties contained by associations. Although both modeling variants are valid, we adhere to the second one as it is more accurate w.r.t. the target specifications of the original annotation type declarations.

Finally, in Figure 5d, the differences regarding the properties of common stereotypes are presented. Except for the JPA profile, we cover all stereotype properties of the RSA profiles. Consequently, our profiles are more complete. The main reason for missing properties in our JPA profile seems to be that RSA provides additional properties for code generation purposes, but these properties are not covered by the JPA library.

Discussion. In this study, we have demonstrated that automatically generated UML profiles from Java libraries comprise a more comprehensive set of stereotypes and features compared to profiles used in practice for the purpose of supporting such libraries. Clearly, the purpose of the developed profiles plays an important role. From a forwardengineering perspective, one may argue that the set of stereotypes, which is actually supported by the accompanying code generators is reasonable to capture at the modeling level. In fact, RSA offers code generation capabilities specific to the profiles we have evaluated in this study. However, for unsupported annotations, which have no corresponding stereotypes, code generators may only produce program code by conventions without allowing developers to intervene in this generation process at the modeling level. From a reverse-engineering perspective, we would lose relevant information at the modeling level if offered profiles provide less capabilities compared to the programming level, which is, however, the case for RSA profiles. Hence, with a fully automated approach, the quality of current profiles can be improved by providing more complete stereotypes that precisely capture the intention of the original annotation types in terms of target definitions, member declarations and return values of such members.

Threats to Validity. There are two main threats that may jeopardize the internal validity of this study. First, we consider only profiles from RSA. The main reason for this procedure is that RSA applies a similar approach as JUMP and offers specific UML profiles for Java libraries. Furthermore, RSA offers standard-compliant UML profiles that conform to the same UML 2 metamodel implementation as used in JUMP. Second, it may be possible that we missed correspondences between elements of the profiles involved in the study. Several kinds of heterogeneities [46] exist that are real challenges 
for model matching algorithms and, thus, may affect the results of our study. However, by applying a two-step matching process which includes a syntactic as well as semantic comparison phase, we tried to minimize the possibility of missing correspondences as a result of different naming conventions and modeling styles. While in the first phase we used a quite conservative matching strategy to avoid false positives, we applied a rather liberal strategy in the second phase to avoid losing potential correspondences.

Concerning external validity, JUMP sets the focus on Java annotations. Many libraries embrace them and real-world cases provide validity for annotated Java code [39]. However, we cannot claim any results outside of Java.

\section{Related Work}

We investigated three lines of research: $(i)$ mappings between Java and UML, $(i i)$ generation of UML profiles and (iii) metamodel generation from programming libraries.

Mapping Java and UML. The elaboration on the mapping between Java and UML has a long tradition in software engineering research $[15,23,28,33]$. Round-trip engineering for UML and Java has been extensively studied in the context of the development of FUJABA [33]. One particular concept of UML that received much attention in the context of Java code generation is the association concept [2,20,21]. However, none of these mentioned approaches consider the transformation of annotation types and their applications from Java to UML. The only exception is the mTurnpike approach [44] that considers Java annotations at the modeling level. Thereby, round-trip transformations between UML models and Java code are realized by considering stereotypes and annotations in the transformations. In contrast, JUMP sets the focus on the automated generation of UML profiles that facilitate round-trip transformations or transformations in general. Besides academic efforts, today's modeling tools support the transformation of Java code to UML models, and vice versa. Their current capabilities and limitations w.r.t. JUMP are discussed in Section 5.1.

Generating $\boldsymbol{U}$ ML Profiles. The only area we are aware of approaches that deal with the automated generation of profiles, is concerned with bridging the gap between MOFbased metamodels and UML's profile mechanism, which is also related to the discussion of an external DSMLs vs. internal DSMLs in UML. Several papers discuss the pros and cons of these approaches(e.g., [42]) and their combination (e.g., [45]). The visualization of domain-specific models in UML with profiles is discussed in [22]. Abouzahra et al. [1] present an approach for interoperability of UML models and DSML models based on mappings between the DSML metamodel and the UML profile. Brucker and Doser [8] go one step further and propose an approach for extending a DSML metamodel for deriving model transformations able to transform DSML models into UML models that are automatically annotated with stereotypes. A related approach is presented in [47], where mappings between the UML metamodel and a DSML metamodel are defined and processed to generate UML profiles for the given DSMLs.

Generating Metamodels. To the best of our knowledge, there is only one automated approach for generating modeling languages from programming libraries-all other automated approaches that deal with exploring libraries, such as [9], set their focus on the generation of domain models rather than a language. API2MoL [11] deals with 
generating metamodels based on Ecore [14] from Java APIs as well as models conforming to the generated metamodels for Java objects instantiated from the Java APIs, and vice versa. As a result, an external Domain-Specific Modeling Language (DSML) is generated from a Java API. While the general idea and motivation of the API2MoL approach is comparable to JUMP, there is a significant difference on how the DSML is realized. JUMP targets UML modelers that are familiar with UML class diagrams and generates internal DSMLs by exploiting the language-inherent extension mechanism of UML, i.e., UML Profiles. Furthermore, annotations are not explicitly considered in the metamodel generation process of API2MoL. One possible reason for neglecting them is that standard versions of current meta-modeling languages, such as Ecore, do not support language-inherent extension mechanisms out-of-the-box [31]. Antkiewicz et al. [3] present a methodology for creating framework-specific modeling languages. While we aim for an automated approach, Antkiewicz et al. use a manual one to create the metamodel and the transformations between model instances and instantiated objects of the frameworks. Again, annotations are not captured by the created languages. When considering the term modeling language in a broader scope, research of related fields consider ontologies as a kind of (meta-)model [19]. In particular, research on ontology extraction from different artifacts is commonly subsumed under the term ontology learning [13]. We are aware of only one approach for extracting ontologies from APIs [40], which neglects, however, also annotations.

To summarize, JUMP is - to the best of our knowledge - the first approach to generate standard-compliant UML profiles from Java libraries that exploit annotations.

\section{Conclusion}

With JUMP, we proposed an approach to close the gap between programming and modeling concerning annotation mechanisms. Thereby, we set the focus on the "Java2UML" case and demonstrated the feasibility of JUMP by generating high-quality UML profiles for numerous Java libraries and applied them in practical reverse-engineering and forward-engineering scenarios. The results gained by our evaluation seem promising. Still, a number of future challenges remain to further integrate programming and modeling. Some interesting differences between Java annotations and UML profiles remain to be explored. On the UML side, inheritance between stereotypes is possible, a concept that is not supported by Java for annotation types. Thus, the design quality of automatically generated UML profiles can be enhanced by exploiting inheritance. On the Java side, retention policies determine at which stages annotations are accessible. UML stereotypes are considered only at design-time. Therefore, an interesting line of future work is to support stereotype applications also during run-time, which becomes especially interesting for executable models, a research area that is currently experiencing its renaissance by the emergence of the FUML standard [34]. Furthermore, we plan to study the support of annotations in other programming languages, e.g., by investigating attributes in C\# and decorators in Python, and how these concepts corresponds to UML profiles. Finally, as we set the focus in this work to platform-specific profiles, we plan to extend this scope to profiles that capture annotations independent of platforms, thereby shifting their application to a more conceptual level. 


\section{References}

1. Abouzahra, A., Bézivin, J., Fabro, M.D.D., Jouault, F.: A Practical Approach to Bridging Domain Specific Languages with UML profiles. In: Proc. Workshop on Best Practices for Model Driven Software Development, pp. 1-8 (2005)

2. Akehurst, D.H., Howells, W.G.J., McDonald-Maier, K.D.: Implementing Associations: UML 2.0 to Java 5. SoSyM 6(1), 3-35 (2007)

3. Antkiewicz, M., Czarnecki, K., Stephan, M.: Engineering of Framework-Specific Modeling Languages. TSE 35(6), 795-824 (2009)

4. Bergmayr, A., Bruneliere, H., Cánovas, J., Gorroñogoitia, J., Kousiouris, G., Kyriazis, D., Langer, P., Menychtas, A., Orue-Echevarria, L., Pezuela, C., Wimmer, M.: Migrating Legacy Software to the Cloud with ARTIST. In: Proc. CSMR, pp. 465-468 (2013)

5. Bergmayr, A., Wimmer, M.: Generating Metamodels from Grammars by Chaining Translational and By-Example Techniques. In: Proc. MDEBE, pp. 22-31 (2013)

6. Brambilla, M., Cabot, J., Wimmer, M.: Model-Driven Software Engineering in Practice. Morgan \& Claypool Publishers (2012)

7. Briand, L.C., Labiche, Y., Leduc, J.: Toward the Reverse Engineering of UML Sequence Diagrams for Distributed Java Software. TSE 32(9), 642-663 (2006)

8. Brucker, A.D., Doser, J.: Metamodel-based UML Notations for Domain-specific Languages. In: Proc. ATEM, pp. 1-15 (2007)

9. Bruneliere, H., Cabot, J., Jouault, F., Madiot, F.: MoDisco: A Generic and Extensible Framework for Model Driven Reverse Engineering. In: Proc. ASE, pp. 173-174 (2010)

10. Canfora, G., Di Penta, M., Cerulo, L.: Achievements and Challenges in Software Reverse Engineering. CACM 54(4), 142-151 (2011)

11. Cánovas, J., Jouault, F., Cabot, J., Molina, J.G.: API2MoL: Automating the Building of Bridges between APIs and Model-Driven Engineering. Information \& Software Technology 54(3), 257-273 (2012)

12. Czarnecki, K., Helsen, S.: Feature-based Survey of Model Transformation Approaches. IBM Systems Journal 45(3), 621-646 (2006)

13. Drumond, L., Girardi, R.: A Survey of Ontology Learning Procedures. In: Proc. WONTO, pp. 1-12 (2008)

14. Eclipse Foundation: Eclipse Modeling Framework, EMF (2014), https://www. eclipse.org/modeling/emf

15. Engels, G., Hücking, R., Sauer, S., Wagner, A.: UML Collaboration Diagrams and their Transformation to Java. In: France, R.B. (ed.) UML 1999. LNCS, vol. 1723, pp. 473-488. Springer, Heidelberg (1999)

16. France, R.B., Bieman, J., Cheng, B.H.C.: Repository for Model Driven Development (ReMoDD). In: Kühne, T. (ed.) MoDELS 2006 Workshops. LNCS, vol. 4364, pp. 311-317. Springer, Heidelberg (2007)

17. France, R.B., Rumpe, B.: The Evolution of Modeling Research Challenges. SoSyM 12(2), 223-225 (2013)

18. Fuentes-Fernández, L., Vallecillo, A.: An Introduction to UML Profiles. Europ. Journal for the Informatics Professional 5(2), 5-13 (2004)

19. Gasevic, D., Djuric, D., Devedzic, V.: Model Driven Engineering and Ontology Development, 2nd edn. Springer (2009)

20. Génova, G., del Castillo, C.R., Lloréns, J.: Mapping UML Associations into Java Code. JOT 2(5), 135-162 (2003)

21. Gessenharter, D.: Mapping the UML2 Semantics of Associations to a Java Code Generation Model. In: Czarnecki, K., Ober, I., Bruel, J.-M., Uhl, A., Völter, M. (eds.) MODELS 2008. LNCS, vol. 5301, pp. 813-827. Springer, Heidelberg (2008)

22. Graaf, B., van Deursen, A.: Visualisation of Domain-Specific Modelling Languages Using UML. In: Proc. ECBS, pp. 586-595 (2007) 
23. Harrison, W., Barton, C., Raghavachari, M.: Mapping UML Designs to Java. In: Proc. OOPSLA, pp. 178-187 (2000)

24. Heidenreich, F., Johannes, J., Seifert, M., Wende, C.: Closing the Gap between Modelling and Java. In: van den Brand, M., Gašević, D., Gray, J. (eds.) SLE 2009. LNCS, vol. 5969, pp. 374-383. Springer, Heidelberg (2010)

25. Jézéquel, J.M., Combemale, B., Derrien, S., Guy, C., Rajopadhye, S.: Bridging the Chasm between MDE and the World of Compilation. SoSym 11(4), 581-597 (2012)

26. Kazman, R., Woods, S.G., Carrière, S.J.: Requirements for Integrating Software Architecture and Reengineering Models: CORUM II. In: Proc. WCRE, pp. 154-163 (1998)

27. Klint, P., Lämmel, R., Verhoef, C.: Toward an Engineering Discipline for Grammarware. ACM Trans. Softw. Eng. Methodol. 14(3), 331-380 (2005)

28. Kollman, R., Selonen, P., Stroulia, E., Systä, T., Zündorf, A.: A Study on the Current State of the Art in Tool-Supported UML-Based Static Reverse Engineering. In: Proc. WCRE, pp. 22-32 (2002)

29. Kolovos, D., Di Ruscio, D., Pierantonio, A., Paige, R.: Different Models for Model Matching: An Analysis of Approaches to Support Model Differencing. In: Proc. CVSM, pp. 1-6 (2009)

30. Kurtev, I., Bézivin, J., Akşit, M.: Technological Spaces: An Initial Appraisal. In: Proc. CoopIS, pp. 1-6 (2002)

31. Langer, P., Wieland, K., Wimmer, M., Cabot, J.: EMF Profiles: A Lightweight Extension Approach for EMF Models. JOT 11(1), 1-29 (2012)

32. Lee, A.: A Scientific Methodology for MIS Case Studies. MIS Quarterly pp. 33-50 (1989)

33. Nickel, U., Niere, J., Zündorf, A.: The FUJABA Environment. In: Proc. ICSE, pp. 742-745 (2000)

34. OMG: FUML (2011), http: / /www . omg . org/ spec / FUML / 1 . 0

35. OMG: MOF (2011), http: / / www .omg.org/spec/MOF

36. OMG: Catalog of UML Profile Specifications (2014), http: / /www . omg . org/spec

37. Oracle: JLS7 (2013), http: / / docs.oracle.com/ javase/specs

38. Pardillo, J.: A Systematic Review on the Definition of UML Profiles. In: Petriu, D.C., Rouquette, N., Haugen, Ø. (eds.) MODELS 2010, Part I. LNCS, vol. 6394, pp. 407-422. Springer, Heidelberg (2010)

39. Parnin, C., Bird, C., Murphy-Hill, E.: Adoption and Use of Java Generics. Empirical Software Engineering 18(6), 1-43 (2012)

40. Ratiu, D., Feilkas, M., Jurjens, J.: Extracting Domain Ontologies from Domain Specific APIs. In: Proc. CSMR, pp. 203-212 (2008)

41. Runeson, P., Höst, M.: Guidelines for Conducting and Reporting Case Study Research in Software Engineering. Empirical Software Engineering 14(2), 131-164 (2009)

42. Selic, B.: The Less Well Known UML: A Short User Guide. In: Proc. SFM, pp. 1-20 (2012)

43. UML-Profile-Store: Project Web Site (2014), http://code.google.com/a/eclipselabs.org/p/uml-profile-store

44. Wada, H., Suzuki, J.: Modeling Turnpike Frontend System: A Model-Driven Development Framework Leveraging UML Metamodeling and Attribute-Oriented Programming. In: Briand, L.C., Williams, C. (eds.) MoDELS 2005. LNCS, vol. 3713, pp. 584-600. Springer, Heidelberg (2005)

45. Weisemöller, I., Schürr, A.: A Comparison of Standard Compliant Ways to Define Domain Specific Languages. In: Giese, H. (ed.) MODELS 2008 Workshops. LNCS, vol. 5002, pp. 47-58. Springer, Heidelberg (2008)

46. Wimmer, M., Kappel, G., Kusel, A., Retschitzegger, W., Schoenboeck, J., Schwinger, W.: Towards an Expressivity Benchmark for Mappings based on a Systematic Classification of Heterogeneities. In: Proc. MDI, pp. 32-41 (2010)

47. Wimmer, M.: A Semi-Automatic Approach for Bridging DSMLs with UML. IJWIS 5(3), 372-404 (2009) 